\title{
The era of research collaborations: new models for working together
}

\author{
Ratko Djukanović ${ }^{1,2}$, Guy Brusselle ${ }^{3}$, Samantha Walker ${ }^{4,5}$, \\ Stephen T. Holgate ${ }^{1,2}$, Sabina Škrgat ${ }^{6}$, Piotr Kuna ${ }^{7}$, Liam G. Heaney ${ }^{8}$, \\ G. Walter Canonica ${ }^{9}$ and Jørgen Vestbo ${ }^{10}$
}

\begin{abstract}
Affiliations: ${ }^{1}$ Clinical and Experimental Sciences, Faculty of Medicine, University of Southampton, Southampton, UK. ${ }^{2}$ NIHR Southampton Respiratory Biomedical Research Unit, Southampton, UK. ${ }^{3}$ Dept of Respiratory Medicine, Ghent University Hospital, Ghent, Belgium. ${ }^{4}$ Asthma UK, London, UK. ${ }^{5}$ European Asthma Research and Innovation Partnership, London, UK. 'University Clinic of Respiratory and Allergic Diseases, Golnik, Slovenia. ${ }^{7}$ Division of Internal Medicine, Asthma and Allergy, Faculty of Medicine, Medical University of Lodz, Lodz, Poland. ${ }^{8}$ Centre for Experimental Medicine, School of Medicine, Dentistry and Biomedical Sciences, Queen's University Belfast, Belfast, UK. ${ }^{9}$ Allergy and Respiratory Diseases Clinic, DIMI Dept of Internal Medicine, University of Genoa - IRCCS San Martino, Genoa, Italy. ${ }^{10}$ Division of Infection, Immunity and Respiratory Medicine, Faculty of Biology, Medicine and Health, University of Manchester, Manchester, UK.
\end{abstract}

Correspondence: Ratko Djukanović, Clinical and Experimental Sciences, mailpoint 810, Level F, Sir Henry Wellcome Laboratories, South Block, Southampton University Hospital, Southampton S016 6YD, UK.

E-mail: rd1dsoton.ac.uk

@ERSpublications

This editorial proposes the establishment of a registry of European centres with expertise in asthma research http://ow.ly/OPHY304Cr9h

Cite this article as: Djukanović $\mathrm{R}$, Brusselle $\mathrm{G}$, Walker $\mathrm{S}$, et al. The era of research collaborations: new models for working together. Eur Respir J 2017; 49: 1601848 [https://doi.org/10.1183/13993003.018482016].

The creation of large national and international collaborations

The past two decades have seen the creation of several European collaborations focusing on asthma and chronic obstructive pulmonary disease (COPD). The growth of the collaboration model for research began in the late 1990s with the Framework Programme (FP)-4-funded project on severe asthma, ENFUMOSA (European Network for Understanding Mechanisms of Severe Asthma), a cross-sectional observational study that provided the proof of principle for large collaborations. Soon after, two more in-depth projects were established, focusing on mechanisms and biomarkers of severe asthma and COPD in two longitudinal cohort studies: BIOAIR (Longitudinal Assessment of Clinical Course and Biomarkers in Severe Chronic Airway Disease) project, funded by FP-5, FP-6 and several national funding bodies; and the GlaxoSmithKline-funded project on COPD, the Evaluation of COPD Longitudinally to Identify Predictive Surrogate Endpoints (ECLIPSE). More recently, the Unbiased Biomarkers in Prediction of Respiratory Disease Outcomes (U-BIOPRED) programme, funded by the European Union (EU) Innovative Medicines Initiative, and the FP-7-funded AirPROM (Airway Disease Predicting Outcomes through Patient Specific Computational Modelling) project have sought to provide a step-change in phenotyping of chronic airways disease through the use of multidimensional clinical outcomes, 'omics biomarkers and sophisticated lung imaging. Indeed, the common ambition of all these projects was to improve the definition of airway disease phenotypes in order to enable better targeting of existing drugs and those in development, and to improve the understanding of determinants of disease severity, disease progression and risk of exacerbations. These collaborations have produced a number of high-impact publications in respiratory journals, like the European Respiratory Journal and the American Journal of

Received: Sept 202016 | Accepted after revision: Sept 202016

Conflict of interest: Disclosures can be found alongside this article at erj.ersjournals.com

Copyright @ERS 2017 
Respiratory and Critical Care Medicine, and major general journals, such as the New England Journal of Medicine and The Lancet, and numerous communications at international meetings. Whilst their direct translation into practical clinical benefit, such as clinical guidelines, is yet to come, they have led to recognition by both the academic and pharmaceutical industry communities that asthma and COPD are not single disease entities. The concept of phenotypes and endotypes is accepted both with respect to mechanisms and selection for treatment with biologics, for example, anti-interleukin-5 antibody for eosinophilic forms of asthma.

During the same period, individual countries have also established national consortia, some focused on asthma [1] and others covering a spectrum of lung diseases: the Netherlands Severe Asthma Research Network, the British Thoracic Society Difficult Asthma Registry, the Belgian Severe Asthma Registry [2], the Spanish Collaboration for Asthma, the German Center for Lung Research, Poland's Polastma (National Programme of Early Diagnosis and Therapy of Asthma) [3], the Finnish National Asthma Programme [4] and the UK National Institutes for Health Research (NIHR) Translational Research Partnership in Inflammatory Respiratory Disease (TRP), all supported by various national public funding schemes. Importantly, the pharmaceutical industry has also signed up to the principle of engaging with multiple investigators, be it in the context of a single company-led project, like ECLIPSE, or collaborations like U-BIOPRED, where as many as 11 large pharmaceutrical companies, members of the European Federation of Pharmaceutical Industries and Associations, four small-to-medium enterprises (SMEs), 20 academic institutions, six patient organisations and asthma charities worked together for 6 years with a bold ambition to speed up drug development in a disease of great unmet health and socioeconomic need. These collaborations have resulted in multimillion-Euro projects, both in basic science and translational research. In some cases, the national consortia submitted joint applications to national funding bodies like the Medical Research Council (MRC) in the UK, awarding multimillion-pound projects like COPD-MAP (MRC and Association of British Pharmaceutical Industry Partnership for COPD), to explore new targets for intervention in COPD, and RASP-UK (Refractory Asthma Stratification Programme in the UK).

So what is it that brings together researchers and, perhaps more intriguingly, academia and multiple companies to work together so intimately on shared objectives? First of all, scientists come together to undertake better science. Several studies have shown that biomedical research that is carried out through international and interdisciplinary collaboration is of significantly higher quality and impact than research performed by a single centre or discipline. The revolution in genetic research is an excellent example to prove the assets of international collaboration. In the 1990s and early 2000s, many candidate-gene studies on a small number of cases and controls were conducted by single research groups, often leading to false-positive results that could not be replicated by other investigators in independent cohorts [5]. Since the late 2000s, however, many genome-wide association studies (using a hypothesis-free approach) have been performed successfully by large international consortia, meta-analysing and replicating the main findings before publishing the robust scientific results. The second reason for working together is that which is of value to all of society: together we are stronger, working together makes hard work easier - "vele handen maken licht werk" as the old Dutch proverb says, "l'unione fa la forza" and "stronger together", the Italian and British equivalents, respectively. Although the scientific and economic impact of these collaborations has not been fully assessed, it stands to reason that pooling scientific expertise, infrastructure and patients enables larger and more complex studies. Thus, the national programmes in Finland, Poland and the UK $[3,4,6]$ focusing on management and education have resulted in marked improvements in clinical outcomes, showing the value of collaborative effort. The U-BIOPRED project on severe asthma [7, 8] applied as many as 10 'omics technologies and knowledge management and data analysis tools [9, 10] for which no single company, academic partner or even country had the required expertise.

How was it that in a climate of industrial and academic competition, partners were willing to work as one, blurring boundaries between the individual pharmaceutical companies and universities? In all cases, the undertaken research was precompetitive, focused on basic science and identification of new targets that all partners could build on and exploit individually. The expense of the large projects of projects like U-BIOPRED has been so high that it would have been unaffordable for individual members, including large multinational pharmaceutical companies.

\section{Setting a joint policy for asthma in Europe}

In 2013, the European Asthma Research and Innovation Partnership (EARIP), comprising members from two pan-European patient organisations, seven academic institutions and two pharmaceutical companies, initiated and coordinated by the UK-based charity, Asthma UK, set out to build on the existing set of individual asthma-related activities across Europe to develop a comprehensive approach to asthma research and to set development priorities, linking basic, translational and clinical science, health service innovation, and drug development. This is the first time that policy makers, researchers, clinicians, 
pharmaceutical and healthcare technology companies, and people with asthma have come together to harmonise the numerous individual efforts in order to enable focus and prioritisation significantly to reduce mortality and morbidity from asthma.

One of EARIP's objectives was to explore ways of improving the delivery of studies, mindful of the rising cost of development of new drugs, coupled with the growing recognition that, with developments in phenotypic stratification of asthma, there was a need to find increasingly smaller populations for trials. A focused working group within EARIP set out to explore the potential for developing a European Research Network of Clinical Asthma Research Facilities (ERNAC), inspired by the NIHR TRP in the UK. The working group conducted an online survey amongst the various stakeholders of asthma research, approaching academics, nonacademic clinicians, nurses, physiotherapists, pharmaceutical companies, clinical research organisations and patients across Europe. The survey comprised questions exploring participants' views about the need for a network, what changes such a network could result in, how it might be managed and funded, what type of research should be the focus of the network, and what were the obstacles to creating and running a successful network. An additional section of the survey sought views about the barriers to development of new treatments for asthma.

\section{Guidance provided by the pan-European asthma research survey: revealing the unmet needs}

Responses were obtained from $20 \mathrm{EU}$ nations, with the best engagement relative to the size of the country coming from Slovenia and the biggest group of respondents being paediatricians. The majority of participants were current researchers and had an interest in clinical research, including drug trials, but around one-third were also engaged in epidemiology, basic science and nonpharmacological research. Although the response was limited to 121 respondents, it nevertheless provided useful insight into the overall potential for a pan-European research network.

Whether or not there is a perceived need for a network was a key question in this survey. When asked whether they thought a "pan-EU network of academic and non-academic clinical centres is needed to make it easier and quicker to do clinical asthma research and shorten the time it takes to develop new drugs for asthma", 91\% responded positively, ranking the need at 4 or 5 on a scale of 0 to 5 . Academic studies funded by public bodies and drug trials not funded by industry came at the top as the type of study that a network would undertake, with industry-funded studies slightly lower. Interestingly, about one-third of respondents were also in favour of studies supported by health insurance companies.

Five general obstacles and challenges were offered for ranking, insufficient funding coming top for one-third of respondents, closely followed by complex EU regulation, with all the other obstacles seen as less important: competition amongst centres, duplication of existing framework and lack of enthusiasm. Only five respondents offered "other" obstacles, such as medical politics and the influence of the pharmaceutical industry, lack of good communication between research centres, coordination and leadership, and poor communication about opportunities. Sustainability was perceived as a greater problem than the initial setting up of a network (67\% versus $33 \%$ of respondents). Competition between centres stood out as a problem for two-thirds of respondents, with only one fifth considering that competition between the various pharmaceutrical companies would be a problem. Finally, respondents felt that obstacles could be overcome with enthusiasm and good funding, $40 \%$ believing that motivation and enthusiasm alone or sufficient funding alone were sufficient. The proposed sources of funding were roughly equally split between governments, grant bodies, charities and universities.

\section{Registry of asthma centres: a good way to start}

Accepting the limitations of the survey, not least under-representation from the pharmaceutical industry, the survey did, in our view, point to considerable enthusiasm for networking. This raises the question of how big the ambition should be and how such a network could be managed. Most respondents (70\%) felt that a network should be affiliated with a society like the European Respiratory Society (ERS). Heartened by the sentiments expressed in the EARIP survey, we propose to start with a realistic and relatively easily achieved ambition to create a registry of European centres with expertise in asthma research. Such a registry could log useful information about the size of the centre, the kind of expertise available in basic, translational and late-phase clinical research, and the numbers of researchers. In order to gauge the level of expertise, each centre would provide a list of its key publications, participation in past and current consortia, and clinical trials. In order to provide a sense of enthusiasm, a measure of readiness to participate in multicentre studies could be given.

What purpose would such a registry serve in the first instance? The registry would be a readily available catalogue for anyone, including the pharmaceutical and biotechnology industries, and diagnostic companies, interested in engaging multiple centres for the purpose of large grant applications and delivery 
of complex clinical trials. Relative to the small effort that would be required, this would provide a good start to a step change in the way research is conducted, and only the future will tell where this could take us. The registry could be a potent vehicle to deliver the ambitions of major international strategiesand guidelines $[11,12]$. If delivered through the ERS Research Agency and the ERS Science Council, this asthma focused registry could test the water for the same models being applied in other respiratory diseases.

\section{Asthma research collaborations: the wider opportunities within the ERS}

Beyond establishing a registry of European centres that have a keen interest in asthma research, there are additional opportunities for asthma researchers (clinicians and scientists) to collaborate within the ERS. First, the ERS provides funding for ERS Clinical Research Collaborations (CRCs), which are pan-European, multicentre networks of principal investigators aiming to advance science and clinical research in a specific respiratory disease area (www.ersnet.org/research/clinical-research-collaborations). The European Multicentre Bronchiectasis Audit and Research Collaboration and the Tuberculosis Network European Trials Group (TBnet), funded by ERS since 2014 and 2015, respectively, are two examples of very successful ERS CRCs in other respiratory domains. More recently, the Severe Paediatric Asthma Collaborative in Europe ERS CRC has been launched, aiming to enhance participation of asthmatic children in therapeutic trials of new biologics and receptor blockers, thus addressing an unmet need raised by paediatricians in the ERNAC survey. Secondly, the ERS offers funding for a whole spectrum of ERS Fellowships (e.g. clinical and research fellowships, as well as fellowships at $\mathrm{PhD}$ level and post-doctoral levels) in order to facilitate and stimulate international mobility and collaboration, as described in a recent editorial by BeLvisi et al. [13]. Lastly, the ERS has established the ERS Research Agency, which among many other scientific activities, offers coordinating and writing activities for consortia of respiratory researchers who strive to obtain external funding at EU level (e.g. Horizon 2020 applications) [14]. In conclusion, as illustrated in this short synopsis of expanding ERS scientific activities, fostering and coordinating international research collaborations in asthma and other respiratory diseases are core activities within the ERS [15].

\section{Acknowledgements}

The authors are very grateful to Judit Varkonyi-Sepp and Blanka Varkonyi for their excellent conduct of the online survey, canvassing views from across Europe about the needs, opportunities and challenges of a European network of asthma research centres.

\section{References}

1 Selroos O, Kupczyk M, Kuna P, et al. National and regional asthma programmes in Europe. Eur Respir Rev 2015; 24: 474-483.

2 Schleich F, Brusselle G, Louis R, et al. Heterogeneity of phenotypes in severe asthmatics. The Belgian Severe Asthma Registry (BSAR). Respir Med 2014; 108: 1723-1732.

3 Kupczyk M, Haahtela T, Cruz AA, et al. Reduction of asthma burden is possible through National Asthma Plans. Allergy 2010; 65: 415-419.

4 Haahtela T, Tuomisto LE, Pietinalho A, et al. A 10 year asthma programme in Finland: major change for the better. Thorax 2006; 61: 663-670.

5 Smolonska J, Wijmenga C, Postma DS, et al. Meta-analyses on suspected chronic obstructive pulmonary disease genes: a summary of 20 years' research. Am J Respir Crit Care Med 2009; 180: 618-631.

6 Gibeon D, Heaney LG, Brightling CE, et al. Dedicated severe asthma services improve health-care use and quality of life. Chest 2015; 148: 870-876.

7 Fleming L, Murray C, Bansal AT, et al. The burden of severe asthma in childhood and adolescence: results from the paediatric U-BIOPRED cohorts. Eur Respir J 2015; 46: 1322-1333.

8 Shaw DE, Sousa AR, Fowler SJ, et al. Clinical and inflammatory characteristics of the European U-BIOPRED adult severe asthma cohort. Eur Respir J 2015; 46: 1308-1321.

9 Wheelock CE, Goss VM, Balgoma D, et al. Application of 'omics technologies to biomarker discovery in inflammatory lung diseases. Eur Respir J 2013; 42: 802-825.

10 Auffray C, Adcock IM, Chung KF, et al. An integrative systems biology approach to understanding pulmonary diseases. Chest 2010; 137: 1410-1416.

11 Reddel HK, Bateman ED, Becker A, et al. A summary of the new GINA strategy: a roadmap to asthma control. Eur Respir J 2015; 46: 622-639.

12 Chung KF, Wenzel SE, Brozek JL, et al. International ERS/ATS guidelines on definition, evaluation and treatment of severe asthma. Eur Respir J 2014; 43: 343-373.

13 Belvisi MG, Morty RE, Rohde G, et al. The ever-expanding ERS fellowship programmes: achievements over the past 3 years. Eur Respir J 2016; 47: 1017-1023.

14 Soriano JB, Paton J, Martin Burrieza F, et al. The ERS Research Agency: the beginning. Eur Respir J 2016; 47: 1017-1023.

15 Migliori GB, Bel E, Joos G, et al. The European Respiratory Society evaluates its 2013-2018 strategic plan implementation. Eur Respir J 2016; 47: 693-698. 